\title{
What Disc Brightness Profiles Can Tell us about Galaxy Evolution
}

\author{
John Beckman ${ }^{1,2,3}$, Peter Erwin ${ }^{4}$ and Leonel Gutiérrez ${ }^{5}$ \\ ${ }^{1}$ Instituto de Astrofísica de Canarias, c/ Vía Lácte a, s/n, E38205, La Laguna, Tenerife, Spain. \\ email: jeb@iac.es \\ ${ }^{2}$ Departamento de Astrofísica. Universidad de La Laguna, Tenerife, Spain. \\ ${ }^{3}$ Consejo Superior de Investigaciones Científicas, Spain. \\ ${ }^{4}$ Max Planck Institut fur Extraterrestrische Physik, Germany \\ ${ }^{5}$ UNAM, Ensenada, México.
}

\begin{abstract}
Azimuthally averaged surface brightness profiles of disc galaxies provide a most useful practical classification scheme which gives insights into their evolution. Freeman (1970) first classified disc profiles into Type I, with a single exponential decline in surface brightness, and Type II, having a split exponential profile, whose inner radial portion is shallower than its outer section. Van der Kruit \& and Searle, (1981) drew attention to sharply truncated profiles of outer discs observed edge-on, but more recently Pohlen et al. (2004) showed that if these same galaxies were observed face-on their profiles would be of Type II. Finally in Erwin, Beckman and Pohlen (2005) we found a significant fraction of profiles with inner portion steeper than the outer portion, which we termed "antitruncations" or Type III profiles. In Erwin, Pohlen and Beckman (2008), we produced a refined classification, taking into account those Type II's produced by dynamical effects at the outer Lindblad resonance, and those Type III's caused by the presence of an outer stellar halo. In Gutiérrez et al. (2011) we showed the distribution of the three main profile types along the Hubble sequence. In early type discs Types I and III predominate, while in late types, Sc and later, Type II predominates.

The evolution of Type II's over cosmic time was studied by Azzollini et al. (2008a, 2008b) who obtained four key results: (a) between $\mathrm{z}=1$ and $\mathrm{z}=0$ the break radius between the inner (shallower) and outer (steeper) profile has increased systematically, by a factor 1.3; (b) the inner profile has steepened while the outer profile is shallower at lower z; (c) the extrapolated central surface brightness has fallen by over two magnitudes; (d) the discs in the full redshift interval are always bluest at the break radius. While this behaviour can be qualitatively explained via evolutionary models including stellar migration plus gas infall, such as that by Roskar et al. (2008), and while Type III profiles may have a qualitative explanation via mergers and/or accretion, the widespread existence of Type I's is still a major conceptual challenge.
\end{abstract}

\section{References}

Azzollini, R., Trujillo, I., \& Beckman, J. E. 2008a, ApJ. Letters 679, L69

Azzollini R., Trujillo, I. \& Beckman, J. E. 2008b, ApJ 684, 1026

Erwin, P. E., Beckman, J. E., \& Pohlen, M. 2005, ApJ. Letters 626, L81

Erwin, J. E., Pohlen, M., \& Beckman, J. E. 2008, AJ 135, 20

Freeman, K. C. 1970, ApJ 160, 767

Van der Kruit, P. \& Searle, L. 1981 A\&SA 95, 105

Gutiérrez, L., Erwin, P., Aladro, R., \& Beckman, J. E. 2011, AJ 142, 145

Pohlen, M., Beckman, J. E., et al. 2004,in: Block, D., et al. (eds.), ASSL, Lecture Notes in Physics (Kluwer, Dordrecht), vol. 317, p. 713

Roskar, R. \& Debattista, P. 2008, ApJ. Letters 675, L65 